\title{
The Conceptual Schema of Ethereum
}

\author{
Antoni Olivé[0000-0001-9806-3007] \\ Department of Service and Information System Engineering \\ Universitat Politècnica de Catalunya - Barcelona Tech, Barcelona, Catalonia \\ antoni.olive@upc.edu
}

\begin{abstract}
There is an abundant literature on Ethereum, but as far as we know what is missing is its explicit conceptual schema. We present here the conceptual schema of Ethereum in UML. The schema should be useful to those that want to understand Ethereum and to those that develop the schema of Ethereum-based DApps. We present a few population constraints, and show that they suffice for the specification at the conceptual level of what is understood by immutability of a blockchain. We also show that the well-known reification construct and an initial constraint suffice to specify at the conceptual level that the Ethereum blockchain stores the full state history.
\end{abstract}

Keywords. Conceptual modeling, Conceptual schema, Blockchain, Ethereum, Immutability.

\section{Introduction}

This paper reports the main results of a project aiming at developing the conceptual schema of Ethereum, a popular open-source platform for blockchain-based decentralized applications [1]. The project had two main goals: (1) to know the conceptual schema of that system, and (2) to check the degree to which the constructs that have been developed in the conceptual modeling field allow the complete specification of a complex system like Ethereum. Of particular concern was how to specify immutability at the conceptual level.

The rationale of the first goal was that so far most of the Ethereum literature is written from either a technical or an economic perspective [2]. Application developers, researchers and students in general that need to learn the foundations of Ethereum have easily available a large number of books, papers and web documents (such as, for example, [3-5]), but they usually include (and, sometimes, focus on) many complex implementation details that make their understanding difficult [6].

From a conceptual modeling point of view, it is easy to see that what is missing in the above literature is the explicit conceptual schema. Ethereum, like all blockchains, is basically a particular kind of distributed database $[7,8]$ and, as such, it necessarily has a conceptual schema. The important role of the explicit definition of that schema not only in the development of database and of information systems, but also in their understanding, has been recognized since long ago [9-11]. 
The rationale of the second goal was that blockchains in general, and Ethereum in particular, have some features whose conceptualization is not obvious. We wanted to check whether the constructs provided by conceptual modeling languages are sufficient to deal with those features. One of them, which is present in all blockchains, is immutability [12]: what kind of integrity constraints are needed to specify immutability? The other feature, which is specific to Ethereum, is that, besides the transactions, it maintains the full state history of the state of the instances of Account, which is the main entity type represented in the blockchain. The question is then: do we need a temporal conceptual model [13-15] to specify the full state history?

We describe here the main result of our project: the conceptual schema of Ethereum in UML. We deal only with the main elements of the structural schema; the behavioral one, at the conceptual level, is simpler. We have found that standard UML, extended with a few known temporal constraints, suffices for defining that schema, including the blockchain immutability and its full state history.

The structure of the paper is as follows. Next section introduces the temporal constraints that will be needed. Section 3 presents the conceptual schema of Ethereum. Section 4 reviews related work. Section 5 briefly summarizes the paper and suggests further work.

\section{Population and initial constraints}

In this section, we define the temporal constraints that will be used in this paper ${ }^{1}$. These constraints have been previously presented in the literature using several terms and formalisms [16-19]. We use here the terminology of the temporal constraints defined in [20] and indicate how to use the constraints as stereotypes in UML.

We assume that entities and relationships are instances of their types at particular time points (or states). By lifespan we mean the set of times during which the system operates. We represent by $E(e, t)$ the fact that $e$ is an instance of entity type $E$ at $t$. We denote by $R\left(p_{1}: E_{1}, \ldots, p_{\mathrm{n}}: E_{\mathrm{n}}\right)$ the schema of a relationship type named $R$ with entity type participants $E_{1}, \ldots, E_{\mathrm{n}}$, playing roles $p_{1}, \ldots, p_{\mathrm{n}}$, respectively. Attributes will be considered as ordinary binary relationship types. We represent by $R\left(e_{1}, \ldots, e_{\mathrm{n}}, t\right)$ the fact that entities $e_{1}, \ldots, e_{\mathrm{n}}$ participate in a relationship instance of $R$ at $t$.

\subsection{Entity type population constraints}

The population of an entity type $E$ is the set of its instances at some time (or state). An entity type is constant when its population is always the same. An entity type $E$ is permanent when once an entity $e$ becomes an instance of $E, e$ continues to be an instance until the end of the lifespan. It can be seen that a constant entity type is also permanent. On the other hand, if $E$ is a covering generalization of a set of permanent entity types, then $E$ is also permanent.

In UML, the above constraints can be defined as stereotyped constraints to which we give the short names of $k$ (for constant) and $p$ (for permanent).

${ }^{1}$ See [23] for the first-order logic formalization of the constraints and examples. 


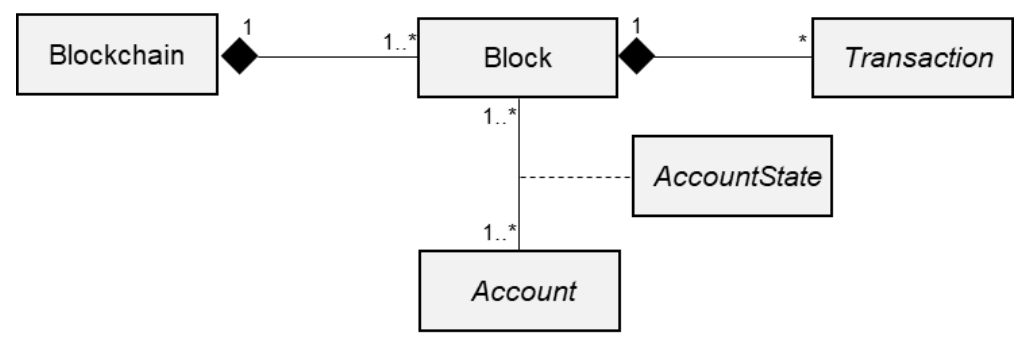

Fig. 1. Main concepts of the conceptual schema of Ethereum

\subsection{Relationship type population constraints}

The population of a relationship type $R$ is the set of its instances (relationships) that exist at some time (or state). We say that a relationship type $R\left(p_{1}: E_{1}, \ldots, p_{\mathrm{n}}: E_{\mathrm{n}}\right)$ is constant with respect to a participant $p_{\mathrm{i}}$ if the instances of $R$ in which an instance $e_{\mathrm{i}}$ of $E_{\mathrm{i}}$ participates are the same during the temporal interval in which $e_{\mathrm{i}}$ exists. Similarly, $R$ is permanent with respect to participant $p$ if the instances of $R$ in which an instance $e_{\mathrm{i}}$ of $E_{\mathrm{i}}$ participates never cease to exist while $e_{\mathrm{i}}$ is an instance of $E_{\mathrm{i}}$.

A relationship type $R$ is constant if it is constant with respect to all its participants. Similarly, $R$ is permanent if it is permanent with respect to all its participants. It can be seen that a constant relationship type is also permanent.

In UML, the above constraints can be defined as stereotyped constraints to which we give the same short names as before: $k$ (for constant) and $p$ (for permanent).

\subsection{Creation-time constraint}

A creation-time constraint $\phi$ of an entity type $E$ is a constraint that its instances must satisfy only at the time when they become an instance of $E$ [21]. Formally:

$\forall e, t\left(\left(E(e, t) \wedge \neg \exists t^{\prime}\left(t^{\prime}<t \wedge E\left(e, t^{\prime}\right)\right) \rightarrow \phi(e, t)\right)\right.$

\section{Ethereum}

Figure 1 is a broad view of the main concepts of the conceptual schema of Ethereum in UML. In the figures, greyed rectangles denote entity types whose complete definition is shown in other figures. The Blockchain consists of a set of Blocks, which in turn consist of a set of Transactions. The state of the system consists of a set of Accounts and their properties. Transactions change that state. For each block, the system stores the state of the accounts (AccountState) at the moment when the transactions included in the block have been processed and the block has been added to the blockchain. In what follows we describe in detail those concepts. 


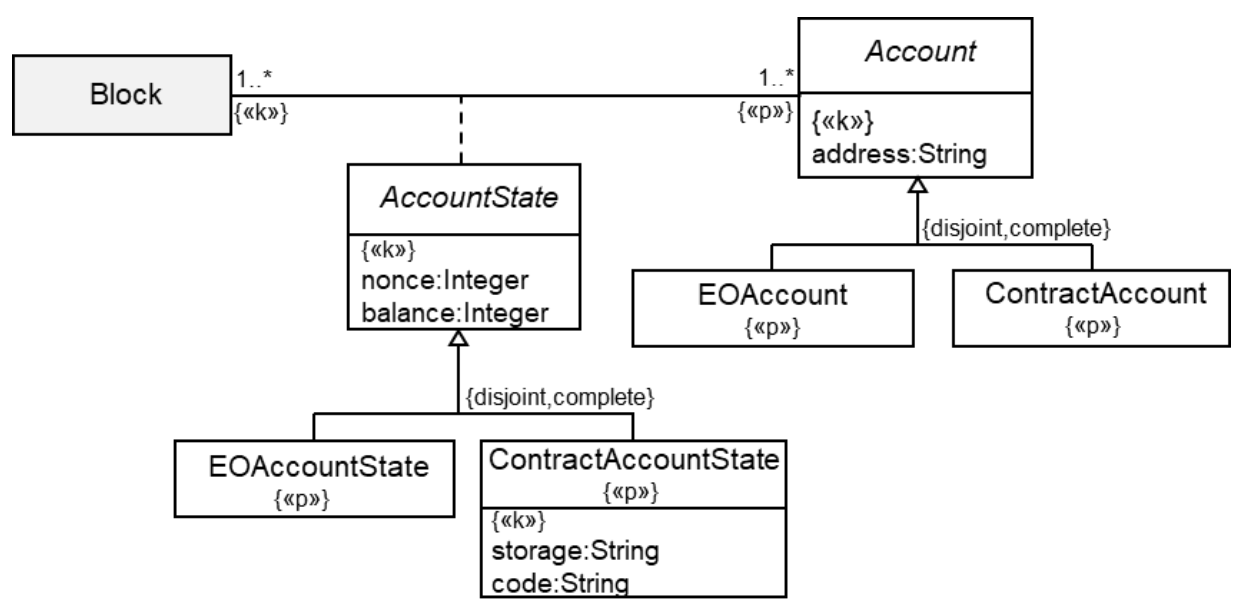

Fig. 2. Accounts and their states in Ethereum

\subsection{Accounts}

There are two kinds of accounts: Externally Owned Account (abbreviated as EOAccount) and ContractAccount, see Fig. 2. Both are permanent. Their generalization is the abstract entity type Account, which is also permanent.

Accounts are identified by means of their address, which is a constant attribute. An externally owned account is created and controlled by a user. Its address is determined from the user public key, which in turn is determined from the user private key. The sets of private/public keys of the users are stored in their wallets (not shown in the Figure).

A contract account is controlled by the code it contains, and its address is assigned by the system when the account is created. A contract account can be created by a user or by the code of another contract account.

Besides their address, both kinds of accounts have two attributes, called nonce and balance. For externally owned accounts, attribute nonce indicates the number of transactions sent from them, while for contract accounts it indicates the number of contract-creations made by them. Attribute balance indicates the amount of ether, the cryptocurrency of Ethereum, owned by the account. The balance is represented in wei, the smallest subunit of ether.

Attributes code and storage apply only to contract accounts. Attribute code contains the code that is executed when called by a transaction or by another contract account. The code is written in the EVM code language, and it is executed by the Ethereum Virtual Machine. Normally, the code of an account cannot change, but there is the possibility of executing a destruct operation with the effect that the code and the storage are removed from the account. Note that if code could not be destructed, then we could define it as a constant attribute of ContractAccount.

Contract accounts have also an attribute called storage, which is used by the contract code. A contract can neither read nor write to any storage apart from its own. 
In Ethereum, there are two kinds of states: world state and account state. An account state is the set of values of the attributes of an account at a given moment. A world state is the set of all accounts existing at a given moment and their account state at that moment.

For each block, Ethereum stores the world state at the moment when the transactions included in a block have been processed and the block has been added to the blockchain. Therefore, the world state of a given block includes all accounts and their state existing after all transactions included in the block have been processed.

The world and the account states have been modeled in Fig. 2 by the association between Block and Account, and its reification, the entity type AccountState. Both the association and AccountState are permanent. In the association, the role block is constant, meaning that the set of accounts to which a block is associated with is fully determined when the block is added to the system, and cannot be changed. The role account in that association is permanent because new instances can be added at any time.

For each block, there is an instance of the association block-account (and therefore of AccountState) for each account that exists at the time the block is created. This can be easily expressed by means of a creation-time constraint (see sect 2.3). In logic, if $R$ is the association block-account, the constraint would be:

$$
\phi(b, t) \equiv \forall a(\operatorname{Account}(a, t) \rightarrow R(b, a, t))
$$

Note that given that Account is permanent, once an account is created, it will be associated with the block within which it was created and with all future blocks.

An instance of AccountState is an account state of the corresponding account. There are two permanent subtypes of AccountState, EOAccountState and ContractAccountState, similarly to the two subtypes of Account. The account attributes have been defined in these entity types, and all of them are constant.

The current values of the account attributes could have been defined as derived attributes of Account and of ContractAcccount. These attributes would not be constant. However, for simplicity, this has not been done in Fig. 2. The derivation rules would indicate that their value is that of the AccountState or ContractAccountState instances corresponding to the same account in the last block.

The set of instances of AccountState of a block is the world state corresponding to that block. Given the population constraints of AccountState, Account, Block (we will see that is also permanent) and those of the association block-account it follows that Ethereum stores the full history of its states.

With respect to immutability, the schema fragment of Fig. 2 indicates that the instances of Account and AccountState cannot be deleted and their attributes cannot be modified. Moreover, the instances of the association block-account of a block cannot be changed. However, and this is a subtle and necessary point, it is possible to add instances of that association to accounts.

\subsection{Transactions}

There are two kinds of transactions: MessageCall and ContractCreation, see Fig. 3. Both are permanent. Their generalization, the abstract entity type Transaction, is also permanent. All attributes of transactions are constant. 


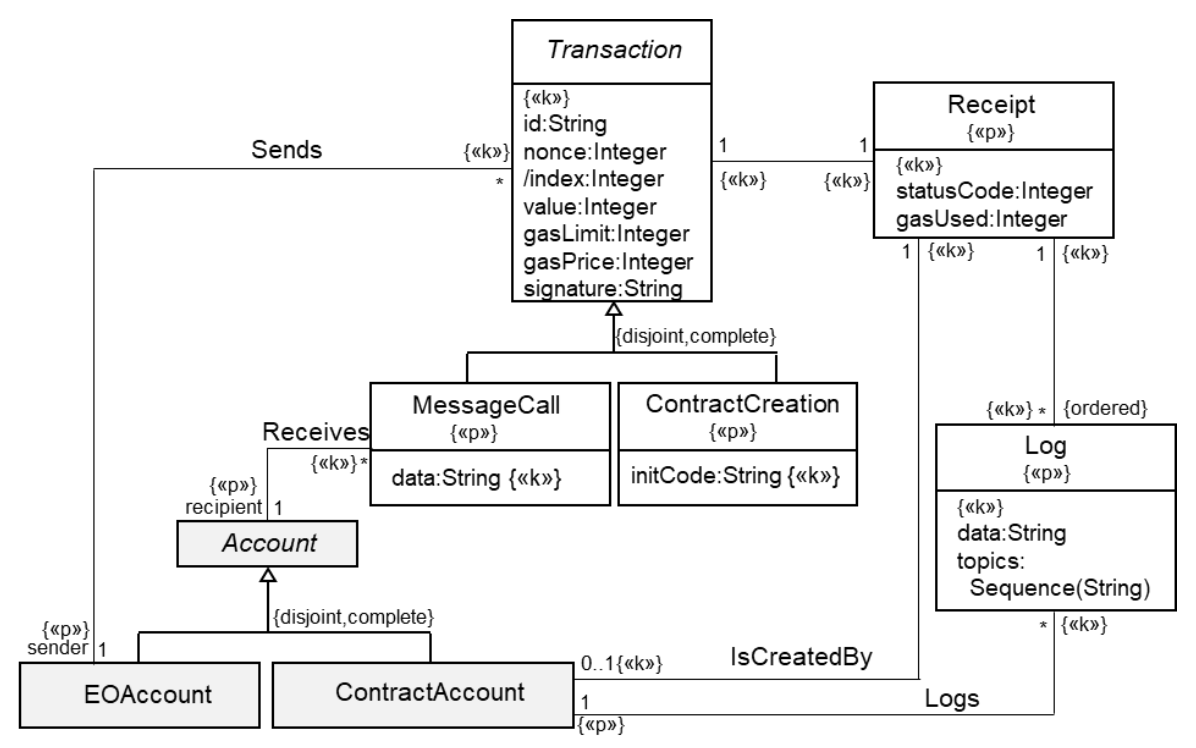

Fig. 3. Transactions in Ethereum

Transactions can be identified in three ways. The first is by means of attribute $i d$, which is automatically computed when the transaction is created. The second is the tuple (sender, nonce). Transactions are originated by externally owned accounts, which send them to the network for processing. The association Sends indicates the sender of a transaction. The association is permanent, with role sender permanent (an account can send several transactions) and role transaction constant (the sender is determined when the transaction is created and cannot be changed later). Transactions sent by an externally owned account are numbered consecutively (nonce) starting at zero. The third way of transaction identification involves attribute index, which will be explained in the next section.

A message call is a transaction sent to a recipient. The association Receives indicates the recipient. The association is permanent with role messageCall constant and role recipient permanent. If the recipient is an externally owned account, the ether indicated in the attribute value is transferred from the sender to the recipient. If the recipient is a contract account, then its code is executed using the transaction attribute data as input. In this case, the transaction value may or may not be transferred to the recipient account.

A contract creation is a transaction that creates a new contract account. The code of the new account is obtained from the initCode attribute and it can be executed in future message calls. The transaction value is the starting balance of the new account (may be zero).

In order to ensure that a transaction has been originated by the sender, the transaction includes a signature attribute. The signature is obtained from the private key of the sender and the transaction attributes. Given a transaction, anyone can check that only the owner of the sender account could have sent it.

In Ethereum, processing a transaction has a fee, which is paid by the sender. The fee is expressed in units of gas. A unit of gas has a price in ether. When the recipient 
of a transaction is a contract account, it may be difficult to know in advance the amount of gas to be spent in a transaction. In order to control the maximum fee to be paid, transactions include attributes gasLimit and gasPrice. The first indicates the maximum number of units of gas to be spent, and the second the price of each unit of gas the sender is willing to pay.

Once a transaction has been executed, Ethereum generates a Receipt that encodes information from the transaction execution. In the association transaction-receipt both roles are constant. Receipt has two constant attributes: statusCode, which indicates whether the transaction has been successful or a failure, and gasUsed, which is the amount of gas used by the transaction. If the transaction was a ContractCreation, then the association IsCreatedBy relates the contract account with the receipt of the transaction that created it. The two roles of the association are constant.

During the execution of a transaction, the code of the contract accounts involved in that transaction can add entries to the log of the transaction. $\log$ is permanent and has two constant attributes: data and topics. The meaning of these attributes is application dependent. The two roles of the association log-receipt are constant. In the Logs association, contractAccount is permanent while log is constant.

With respect to immutability, the schema fragment of Fig. 3 states that the instances of Transaction, and its subtypes, Receipt and Log cannot be deleted and their attributes cannot be modified. Three associations (transaction-receipt, logreceipt and $I s C r e a t e d B y$ ) are constant, meaning that their instances cannot be deleted and no new instances can be added to the existing participants in those associations. The other three associations (Sends, Receives, Logs) are permanent, which implies that their instances cannot be deleted, but it is possible to add new instances to entities with a permanent role.

\subsection{Blocks}

In Ethereum, the blockchain consists of an ordered sequence of blocks. Figure 4 shows the entity types Blockchain and Block and the composition association between them. Blockchain is constant, and its population consists of a single instance, while Block is permanent and its population consists of many instances. The role blockchain is permanent because new blocks are added to the composition, while the role block is constant because a block is associated to the blockchain when it is created and cannot be changed.

All attributes of Block are constant. The first two are identifiers of blocks. Attribute $i d$ is a hash computed by the system from the block's contents, which includes several attributes irrelevant to our conceptual modelling purposes. Attribute number is derived. The corresponding derivation rule defines its value as the index of the block in the composition. The first block has a number of zero.

As has been indicated, the role block in the blockchain composition is constant. However, in this case the role block is ordered, which means that the blocks of the blockchain are ordered (a sequence in this case). This raises a subtle point: what precludes the change of the order of the blocks in the sequence? We could define a new population constraint for this purpose but in this case it is not necessary. It 


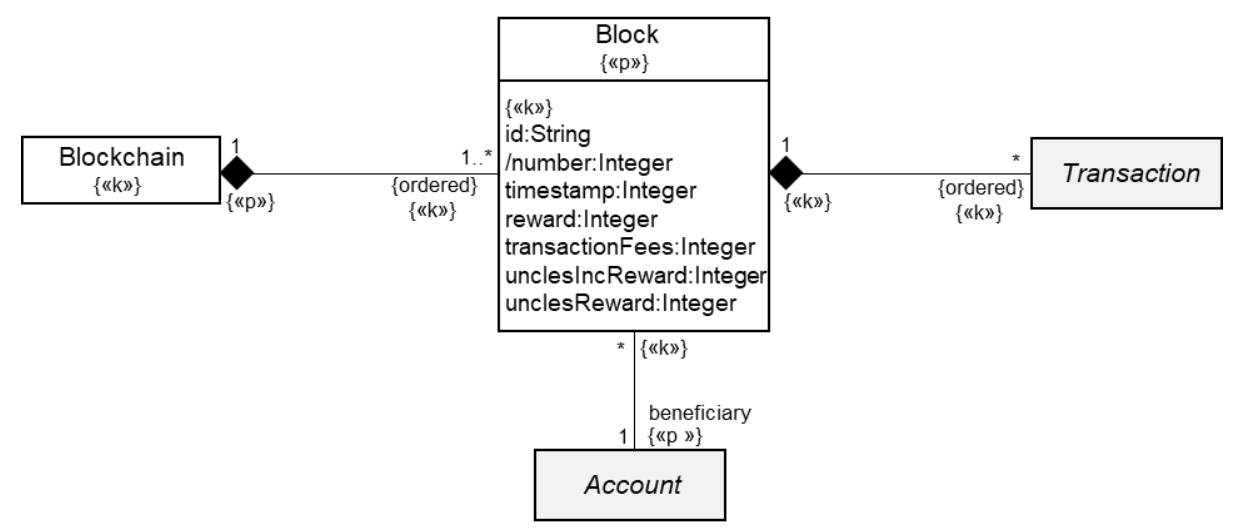

Fig. 4. Blocks in Ethereum

suffices to define attribute number as constant, which implies that the position of a block in the sequence cannot change.

Attribute timestamp indicates the time when the block was added to the blockchain. An obvious constraint is that it must be greater than that of the previous block in the sequence.

Blocks are prepared and added to the blockchain by miners, which are specialized network nodes. The miner of a block is compensated (in ether) for the work done. The compensation is sent to an account designated by the miner, given by the association block-beneficiary in Fig. 4. The compensation includes a reward (attribute reward) and the fees of all transactions included in the block (attribute transactionFees). In some cases, a block may include up to two special stale blocks, called uncle blocks, which do not include transactions. If it is so, then the beneficiary receives an additional reward (attribute unclesIncReward), and the uncles receive the reward given by attribute unclesReward. For simplicity, Figure 4 shows neither the uncle blocks nor their beneficiaries.

A block, in turn, consists of an ordered sequence of transactions. Figure 4 shows the composition association between Block and Transaction. Note that both roles in that association are constant: the instances of the composition are determined when a block and a transaction are created and cannot be changed. A block and its transactions are recorded in the blockchain at the same time.

In addition to the two ways indicated in the previous section, an instance of Transaction can be identified by the block of which it is a part and the index attribute (Fig. 3). This is a derived attribute whose value is the position of the transaction in the block. The attribute is constant, which -among other things- means that the position on a transaction in the block cannot be changed.

With respect to immutability, the schema fragment of Fig. 4 indicates that the single instance of Blockchain exists since the beginning of the system's lifespan and, as well as the instances of Block, it cannot be deleted. The attributes of both types cannot be changed. One association (block-transaction) is constant. The other two are permanent. It is possible to add blocks to the blockchain (association blockchainblock) and to add blocks to an account (association block-beneficiary). Changing the 
position of a block in the blockchain or the position of a transaction in a block is not allowed.

It is interesting to see that the population constraints allow us to easily define that it is possible to add blocks to the blockchain, but that it is not possible to add transactions to a block.

\section{Related work}

In the literature, the two works that are more related to ours are the blockchain domain ontology [2] and EthOn [6,22]. The blockchain domain ontology is not blockchain-specific, but general. It distinguishes three ontological layers (datalogical, infological and essential) and it includes an ontology for each layer. Our conceptual schema would basically be placed in their infological layer. The ontology corresponding to this level, the infological ontology, consists of six entity types, five associations, and one attribute. The conceptual schema that we have presented here is much more detailed because it is blockchain-specific.

EthOn is an ontology in RDF Schema and OWL that formalizes most of the concepts used in the Ethereum platform as described in the "yellow paper" [1]. The scope of EthOn is different from that of our conceptual schema. EthOn includes in an integrated ontology both the concepts related to the data stored in the platform and the concepts related to the implementation. In the classical terminology used in conceptual modelling [9], it can be said that EthOn describes in an integrated view both the conceptual and the internal schema of Ethereum. On the other hand, EthOn does not specify the population constraints of its concepts needed to specify their immutability, and it does not formalize the full state history.

\section{Conclusions}

We have presented the conceptual schema of Ethereum in UML. As far as we know, this is the first time that the schema is presented in the literature. We hope the schema will be useful to those that want to understand Ethereum and to those that develop the schema of Ethereum-based DApps [23].

We have presented and formalized a few population constraints, and we have shown that they suffice for the specification at the conceptual level of what is understood by immutability of a blockchain. Finally, we have shown that the wellknown reification construct and an initial constraint suffice to specify at the conceptual level that the Ethereum blockchain stores the full state history.

This work can be extended in several directions. We point out two of them here. First, it would be useful to complete the structural schema that we have presented with a few remaining details, and to develop the behavioral one. Second, a work similar to the one presented here could be done with other blockchain platforms.

Acknowledgments. The author is greatly indebted to Joan Antoni Pastor and Jordi Estapé for their comments to earlier drafts of this paper. 


\section{References}

1. Wood, G. Ethereum: A secure decentralised generalised transaction ledger, https://ethereum.github.io/yellowpaper/paper.pdf (2020).

2. de Kruijff J., Weigand H.: Understanding the Blockchain Using Enterprise Ontology. In: Dubois E., Pohl K. (eds.) Advanced Information Systems Engineering. CAiSE 2017. LNCS, vol. 10253 (2017)

3. Antonopoulos, A.M., Wood, G.: Mastering Ethereum: Building Smart Contracts and DApps. O'Reilly Media (2018)

4. Dameron, M.: Beigepaper: An Ethereum technical specification. https://github.com/chronaeon/beigepaper/blob/master/beigepaper.pdf (2019)

5. Kasireddy, P.: How does Ethereum work, anyway? https://medium.com/@ preethikasireddy/ how-does-ethereum-work-anyway-22d1df506369 (2017)

6. Pfeffer, J.: EthOn - introducing semantic Ethereum. Organized Ethereum knowledge, https://media.consensys.net/ethon-introducing-semantic-ethereum-15f1f0696986 (2017)

7. Dinh, T.T.A., Liu, R., Zhang, M., Chen, G., Ooi, B. C., Wang, J.: Untangling Blockchain: A Data Processing View of Blockchain Systems, in IEEE TKDE, 30(7), pp. 1366-1385 (2018)

8. Kim, H.M., Laskowski, M.: Toward an ontology-driven blockchain design for supply-chain provenance. Int. Syst. in Accounting, Finance and Management 25(1): 18-27 (2018)

9. ANSI: ANSI/X3/SPARC study group on data base management systems. Interim report. FDT, Bulletin of ACM SIGMOD 7(2) (1975)

10. Mylopoulos, J.: Conceptual Modelling and Telos. In: Loucopoulos, P, Zicari, R. (eds.) Conceptual Modelling, Databases and CASE, John Wiley and Sons, pp. 49-68. (1992)

11. Delcambre L.M.L., Liddle S.W., Pastor O., Storey V.C.: A Reference Framework for Conceptual Modeling. In: Trujillo J. et al. (eds.) Conceptual Modeling. ER 2018. LNCS, vol. 11157 (2018)

12. Hofmann, F., Wurster, S., Ron, E., Böhmecke-Schwafert, M.: The immutability concept of blockchains and benefits of early standardization, 2017 ITU Kaleidoscope: Challenges for a Data-Driven Society (ITU K), Nanjing, pp. 1-8. (2017)

13. Gregersen, H., Jensen. C.S.: Temporal entity-relationship models-a survey, IEEE TKDE, vol. 11, no. 3, pp. 464-497 (1999)

14. Combi C., Degani S., Jensen C.S.: Capturing Temporal Constraints in Temporal ER Models. In: Li Q. et al. (eds.) Conceptual Modeling - ER 2008. LNCS, vol. 5231. (2008)

15. Artale A., Franconi E.: Foundations of Temporal Conceptual Data Models. In: Borgida A.T., Chaudhri V.K., Giorgini P., Yu E.S. (eds.) Conceptual Modeling: Foundations and Applications. LNCS, vol. 5600. Springer, (2009)

16. Costal, D., Olive, A., Sancho, M.R.: Temporal features of class populations and attributes in conceptual models. In: Embley, D.W. (ed.) ER 1997. LNCS, vol. 1331, pp. 57-70. (1997)

17. Cabot J., Olivé A., Teniente E.: Representing Temporal Information in UML. In: Stevens P., Whittle J., Booch G. (eds.) «UML» 2003 - The Unified Modeling Language. Modeling Languages and Applications. LNCS, vol. 2863. (2003)

18. Artale, A., Parent, C., Spaccapietra, S.: Evolving objects in temporal information systems. Ann. Math. Artif. Intell. 50(1-2): 5-38 (2007)

19. McBrien P.: Temporal Constraints in Non-temporal Data Modelling Languages. In: Li Q. et al. (eds.) Conceptual Modeling-ER 2008. LNCS, vol. 5231. (2008)

20. Olivé, A.: Conceptual Modeling of Information Systems. Springer, Berlin (2007)

21. Olivé, A.: A method for the definition of integrity constraints in object-oriented conceptual modeling languages. Data Knowl. Eng. 59(3): 559-575 (2006)

22. Pfeffer, J.: EthOn: An Ethereum Ontology. https://consensys.github.io/EthOn/ EthOn_spec.html. Accessed (March 2020)

23. Olivé, A.: The conceptual schema of Ethereum and of the ERC-20 token standard. http://hdl.handle.net/2117/328036 (2020) 